\title{
Magnetic Properties of Transition Metal Molybdates
}

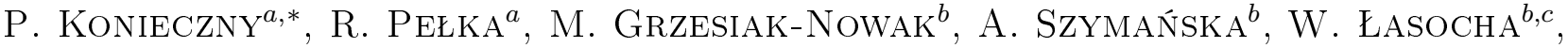 T. WASIUTYŃSKI ${ }^{a}$}

${ }^{a}$ Department of Structural Research, Institute of Nuclear Physics, Polish Academy of Science,

Radzikowskiego 152, 31-342 Kraków, Poland

${ }^{b}$ XRD and Thermoanalysis Laboratory, Jerzy Haber Institute of Catalysis and Surface Chemistry Polish Academy of Science, Niezapominajek 8, 30-239 Kraków, Poland

${ }^{c}$ Faculty of Chemistry, Jagiellonian University, Ingardena 3, 30-060 Kraków, Poland

Two examples of transition metal molybdates were studied with the use of X-ray diffraction and DC magnetometry techniques. First compound, copper dimolybdate trihydrate $\mathrm{CuMo}_{2} \mathrm{O}_{7} \cdot 3 \mathrm{H}_{2} \mathrm{O}$ contains $2 \mathrm{D}$ layers. The second one is fibrillar cobalt trimolybdate octahydrate $\mathrm{CoMo}_{3} \mathrm{O}_{10} \cdot 8 \mathrm{H}_{2} \mathrm{O}$. Both compounds exhibit paramagnetic behaviour at room temperature. However, in the first one copper ions form dimers linked by oxygen bridges, in which exchange interactions between two localized spin states occur. The second compound consists of isolated cobalt ions in the octahedral surroundings, resulting in magnetic properties deviating from the Curie law.

DOI: $10.12693 /$ APhysPolA.126.250

PACS: 75.50.Xx, 75.50.Ee, 75.50.-y

\section{Introduction}

In this work we report our joint experimental efforts to understand the behavior of $\mathrm{CuMo}_{2} \mathrm{O}_{7} \cdot 3 \mathrm{H}_{2} \mathrm{O}$ and $\mathrm{CoMo}_{3} \mathrm{O}_{10} \cdot 8 \mathrm{H}_{2} \mathrm{O}$, henceforth abbreviated as $\left\{\mathrm{CuMo}_{2}\right\}$ and $\left\{\mathrm{CoMo}_{3}\right\}$.

The space group of $\left\{\mathrm{CuMo}_{2}\right\}$ is $\mathrm{P}-1$ with lattice parameters $a=8.678(7) \AA, b=9.289(4) \AA, c=6.064(5) \AA$, $\alpha=90.72(9)^{\circ}, \beta=95.99(5)^{\circ}, \gamma=97.42(8)^{\circ}$, and for $\left\{\mathrm{CoMo}_{3}\right\}$ the space group is $\mathrm{P} 2_{1} / \mathrm{c}$ with lattice parameters $a=12.072(3) \AA, b=19.833(2) \AA, c=7.624(9) \AA$, $\beta=107.58(5)^{\circ}$. The first compound contains twodimensional layers of dimolybdate chains interconnected by $\left[\mathrm{Cu}_{2} \mathrm{O}_{4}\left(\mathrm{H}_{2} \mathrm{O}\right)_{6}\right]^{4+}$ (Fig. 1). Copper ions with spin $S_{C u}=1 / 2$ are grouped in isolated pairs forming dimers.

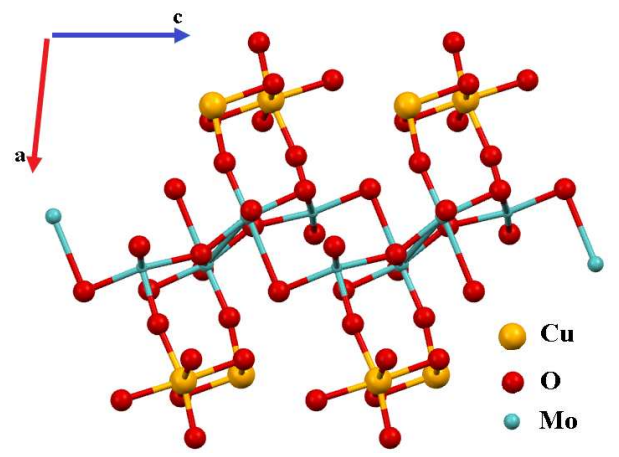

Fig. 1. View of the structure $\left\{\mathrm{CuMo}_{2}\right\}$ along the $\boldsymbol{b}$ crystallographic axis. The water molecules are not shown for clarity.

The second compound consists of zigzag anionic chains of $\mathrm{Mo}_{3} \mathrm{O}_{10}^{2-}$ which are separated by $\left[\mathrm{Co}\left(\mathrm{H}_{2} \mathrm{O}\right)_{6}^{2+}\right]$ and wa-

*corresponding author; e-mail: piotr.konieczny@ifj.edu.pl ter molecules (Fig. 2). In this sample Co ions are in almost perfect octahedral geometry, with spin $S_{C o}=3 / 2$. Molybdenum in both cases is $\mathrm{Mo}^{+6}$ with spin $S_{M o}=0$.

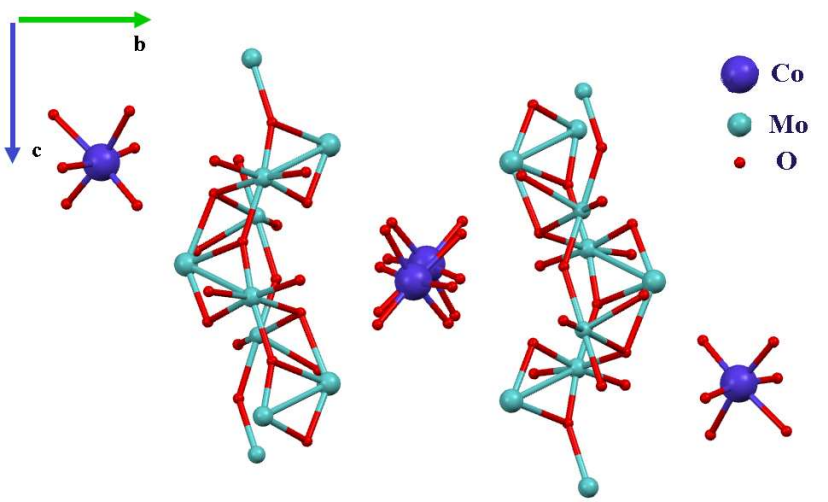

Fig. 2. Structure of $\left\{\mathrm{CoMo}_{3}\right\}$ along the $\boldsymbol{a}$ crystallographic axis. The water molecules are not shown for clarity.

\section{Experimental}

The polycrystalline samples of $\left\{\mathrm{CuMo}_{2}\right\}$ and $\left\{\mathrm{CoMo}_{3}\right\}$ were synthesized according to the methods reported in [1]. X-ray powder diffraction patterns for both compounds were collected on a Philips X'pert Pro diffractometer in transmission mode.

The Quantum Design SQUID MPMS-XL magnetometer was used to measure the isothermal magnetization at $T=4 \mathrm{~K}$ in the field range of $[-55,55] \mathrm{kOe}$ and the static magnetic susceptibility $\chi_{d c}$ in the temperature range of $2.0-300 \mathrm{~K}$ and in the applied field of 500 Oe.

\section{Results and discussion}

Figure 3 presents the dc susceptibility for $\left\{\mathrm{CuMo}_{2}\right\}$ in terms of $\chi \cdot T$ as a function of temperature. In the case of an ordinary paramagnetic compound this type of plot should show a linear behaviour with constant 
value, which is the Curie constant. However this nonlinear shape of the curve in Fig. 3 is characteristic for dinuclear compounds with isotropic interactions [2]. $\mathrm{Cu}^{2+}$ ions in $\left\{\mathrm{CuMo}_{2}\right\}$, each with $S_{C u}=1 / 2$, form pairs which are linked by $\mathrm{O}^{2-}$. Those two local doublet states interact with each other, which causes that the total spin of $S_{C u-C u}=1$ or $S_{C u-C u}=0$ is a good quantum number. Moreover the decrease of the $\chi \cdot T$ values at low temperatures apparent in Fig. 3 suggests an antiferromagnetic interaction [2].

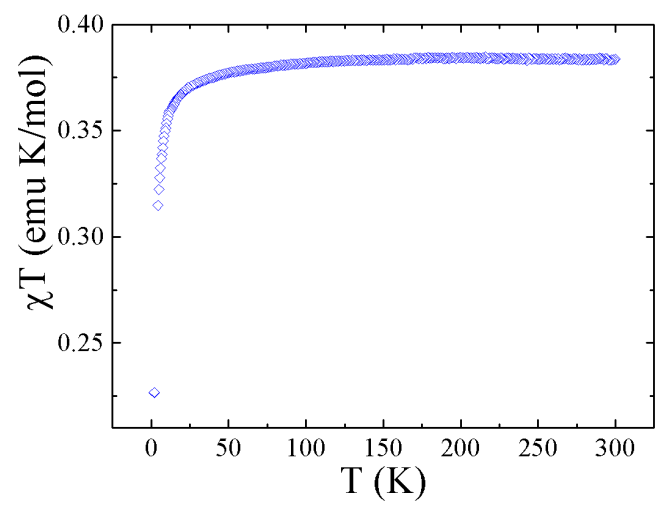

Fig. 3. $\chi \cdot T$ as a function of temperature with $H_{d c}=$ 500 Oe for $\left\{\mathrm{CuMo}_{2}\right\}$.

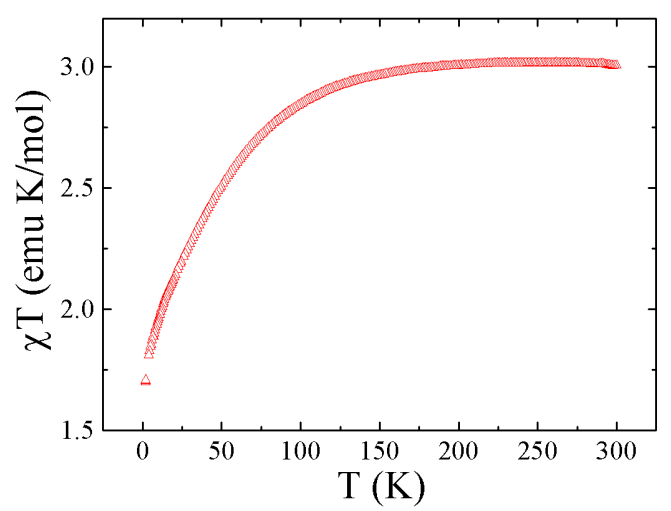

Fig. 4. $\chi \cdot T$ as a function of temperature with $H_{d c}=$ 500 Oe for $\left\{\mathrm{CoMo}_{3}\right\}$.

In the case of $\left\{\mathrm{CoMo}_{3}\right\}$ the $\mathrm{Co}^{2+}$ ions do not interact with each other, however the shape of the $\chi \cdot T$ plot (Fig. 4) is also far from the Curie law type. This is due to the nonvanishing zeroth-order contribution of the orbital angular momentum to the total magnetic moment of the ion.

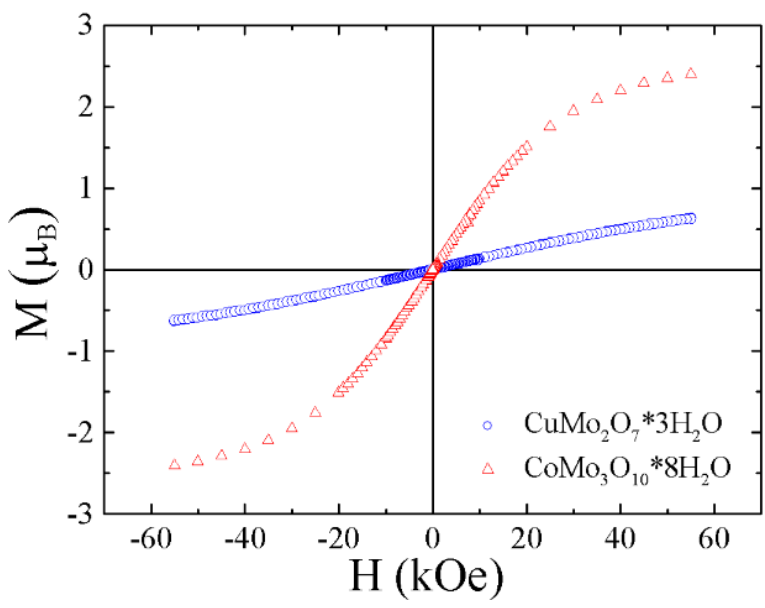

Fig. 5. Magnetization as a function of applied field at $T=4.0 \mathrm{~K}$ for both compounds.

The results of isothermal magnetization for both compounds at $T=4.0 \mathrm{~K}$ can be seen in Fig. 5 . It can be noticed that for both compounds the magnetization fails to saturate in the field of $55 \mathrm{kOe}$. The saturation values of the magnetization, $M_{s a t}=g \mu_{B} S$, extrapolated to the infinite magnetic field for $\left\{\mathrm{CuMo}_{2}\right\}$ is $1.1 \mu_{B} /$ f.u. which agrees with $S_{C u}=1 / 2$ and $g \approx 2.2$. As for $\left\{\mathrm{CoMo}_{3}\right\}$, where Co is high spin with $S_{C o}=3 / 2$ and $L=2$, the case is more complicated due to spin-orbit coupling and needs further analysis.

\section{Conclusions}

Magnetic measurements of two metal molybdates revealed the deviations from the paramagnetic Curie law. In the case of $\left\{\mathrm{CuMo}_{2}\right\}$ the $\chi \cdot T$ curve indicates the intramolecular antiferromagnetic interaction in the dimer of copper ions. The $\chi \cdot T$ signal of the $\left\{\mathrm{CoMo}_{3}\right\}$ compound evidences a structure of quantum substates, spanning a broad temperature interval due to crystal-field and spinorbit coupling.

\section{Acknowledgments}

P.K., M.G.-N. and A.S. have been partly supported by the EU Human Capital Operation Program, Polish Project No. POKL.04.0101-00-434/08-00.

\section{References}

[1] B. Gawel, W. Surga, W. Lasocha, Zeitschrift für Kristallographie Supplements 30, 381 (2009).

[2] O. Kahn, Molecular Magnetism, VCH Publishers, 1993, p. 103.

[3] O. Kahn, Molecular Magnetism, VCH Publishers, 1993, p. 38. 